\title{
Effect of Error Field Correction Coils on W7-X Limiter Loads
}

\author{
S.A. Bozhenkov ${ }^{1}$, M.W. Jakubowski ${ }^{1}$, H. Niemann ${ }^{1}$, \\ S.A. Lazerson ${ }^{2}$, G.A. Wurden ${ }^{5}$, C. Biedermann ${ }^{1}$, G. Kocsis ${ }^{3}$, \\ R. König ${ }^{1}$, F. Pisano ${ }^{6}$, L. Stephey ${ }^{4}$, T. Szepesi ${ }^{3}$, U. Wenzel ${ }^{1}$, \\ T.S. Pedersen ${ }^{1}$, R.C. Wolf ${ }^{1}$, W7-X team \\ 1 Max-Planck-Institut für Plasmaphysik, Greifswald, Germany \\ 2 Princeton Plasma Physics Laboratory, Princeton, NJ, USA \\ 3 Wigner Institute, Budapest, Hungary \\ ${ }^{4}$ University of Wisconsin, Madison, WI, USA \\ ${ }^{5}$ Los Alamos National Laboratory, Los Alamos, NM, USA \\ ${ }^{6}$ University of Cagliari, Italy \\ E-mail: sergey.bozhenkov@ipp.mpg.de
}

\begin{abstract}
In the first campaign Wendelstein 7-X was operated with five poloidal graphite limiters installed stellarator symmetrically. In an ideal situation the power losses would be equally distributed between the limiters. The limiter shape was designed to smoothly distribute the heat flux over two strike lines. Vertically the strike lines are not uniform because of different connection lengths. In this paper it is demonstrated both numerically and experimentally that the heat flux distribution can be significantly changed by non-resonant $n=1$ perturbation field of the order of $10^{-4}$. Numerical studies are performed with field line tracing. In experiments perturbation fields are excited with five error field trim coils. The limiters are diagnosed with infrared cameras, neutral gas pressure gauges, thermocouples and spectroscopic diagnostics. Experimental results are qualitatively consistent with the simulations. With a suitable choice of the phase and amplitude of the perturbation a more symmetric plasma-limiter interaction can be potentially achieved. These results are also of interest for the later W7-X divertor operation.
\end{abstract}

\section{Introduction}

Wendelstein 7-X is a helical axis optimized superconducting stellarator [1]. For steady state operation W7-X will be equipped with a water-cooled island divertor and a water-cooled graphite armor of the endangered parts of the first wall [2, 3]. In the first experimental campaign, that took place from December 2015 till March 2016, the machine was instead operated with poloidal limiters [4]. Plasma break-down and heating were provided by Electron Cyclotron Resonance Heating (ECRH) with the total power of up to $4.3 \mathrm{MW}$. Electron temperatures of about $7 \mathrm{keV}$, ion temperatures of about $2 \mathrm{keV}$ and electron densities of about $4 \cdot 10^{19} \mathrm{~m}^{-3}$ were achieved simultaneously, plasmas of up 


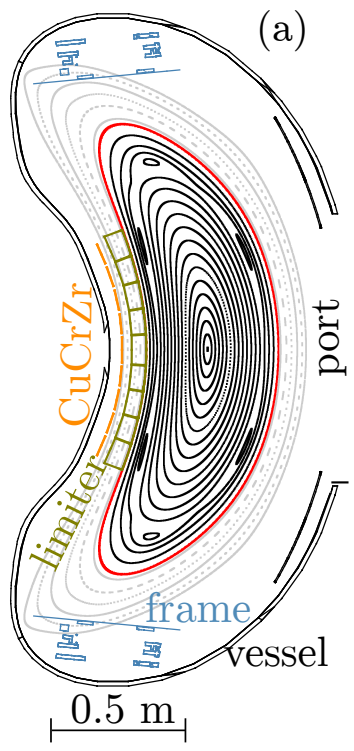

(b)

(c)
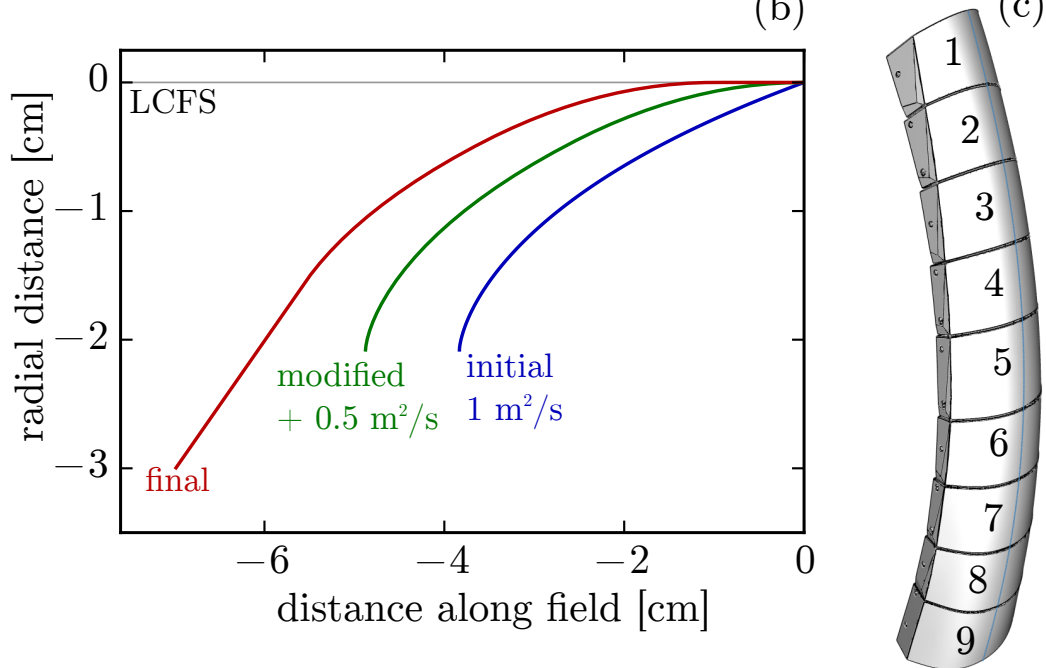

Figure 1. Limiter configuration and limiter shape. (a) - Poincaré plot of the primary limiter configuration in $\varphi=0$ plane. The last closed flux surface is plotted in red. Main in-vessel components are indicated. (b) - limiter shape in a plane parallel to the field. As the heat flux density decays in the scrape-off layer, steeper incidence angles of the field are allowed. Line "initial" (blue) is the solution of equation 1 with $D_{\perp}=1 \mathrm{~m}^{2} / \mathrm{s}$. Line "modified" (green) is the modified shape to allow $D_{\perp}=0.5 \mathrm{~m}^{2} / \mathrm{s}$ and hydrogen operation. Line "final" (red) is the final design obtained by inserting a plateau in the center and a linear part at the edge. (d) - 3d shape of the inboard limiter and numbering of the tiles.

to $6 \mathrm{~s}$ were sustained [5]. In this paper shape of limiters, expected power distribution and influence of $1 / 1$ error field on the limiter power distribution are discussed. An intentionally superimposed error field can be potentially used to counteract limiter misalignments.

In the first campaign the plasma was limited by five identical limiters installed stellarator symmetrically in the bean shaped planes, one limiter per W7-X module. In an ideal situation the power losses would be equally distributed between the limiters. The heat flux distribution can become asymmetric due to limiter installation errors. In this paper it is demonstrated that the heat flux distribution can be also significantly changed by non-resonant $1 / 1$ perturbation field of the order of $10^{-4}$, where the error field is intentionally superimposed. Both numerical and experimental results are presented. Numerical studies are performed with field line tracing [6]. In the experiments the perturbation fields are superimposed with a set of five error field trim coils [7], and the limiters are observed with a set of diagnostics. These results are also of interest for the later W7-X divertor operation, where similar questions will have to be addressed.

The rest of the paper is organized in the following way: first in section 2 the limiter design and the expected ideal heat flux patterns are explained; in section 3 the influence of radial perturbation fields is explored numerically; then in section 4 results of the experiments with trim coils are presented; and finally in section 5 a summary of the 
results is given.

\section{Poloidal inboard limiter}

In the first operational phase most of the graphite armor components and the island divertor were not installed. To protect metal parts, in particular divertor frame structure and the $\mathrm{CuCrZr}$ tiles to be covered later with graphite, and to guarantee a reasonable performance, a limiter configuration was used. Five graphite limiters, matching the fivefold machine symmetry, were installed in symmetry planes $\varphi=0$ at the inboard side, figure 1 1 a. The radial position of the limiters was chosen to restrict the power reaching the first wall to below $1 \%$.

Because of the wall safety, only a limited number of magnetic configurations without large islands in the scrape-off layer was used in experiments. The primary limiter configuration shown in figure 1 $1 \mathrm{a}$ is obtained with the currents in the planar coils of 0.13 relative to that in the non-planar coils. At W7-X, the non-planar coils generate the confining magnetic field, whereas the planar coils can be used to adjust both the rotational transform and the horizontal position. Further details of the W7-X magnetic system and of specifying magnetic configurations can be found in [11]. The rotational transform for the configuration used here is about 0.79 in the center and 0.87 at the edge [4]. The effective radius of the last closed flux surface (LCFS) is about $0.49 \mathrm{~m}$. A $m / n=6 / 5$ island chain is present at the edge of the confinement region, but does not compromise the safety of the first wall.

The shape of the limiters was designed to approximate a uniform distribution of the heat flux in a plane parallel to the field [8]. As the heat flux density decays with the distance to the LCFS, steeper incidence angles of field lines are allowed:

$$
F_{0} \cdot e^{y / \lambda} \cdot \sin \alpha=\Phi
$$

Here $F_{0}$ is the parallel heat flux density at the LCFS, $\alpha(y)$ is the angle between field lines and the limiter surface with $y^{\prime}=\tan \alpha, y$ is the distance from the LCFS (negative) and $\Phi=10 \mathrm{MW} / \mathrm{m}^{2}$ is the design value of heat flux density to the limiter. The perpendicular decay length at the limiter position was estimated to be about $1.5 \mathrm{~cm}$ for helium discharges with $D_{\perp}$ of $1 \mathrm{~m}^{2} / \mathrm{s}$ and $T_{i}$ of about $100 \mathrm{eV}$. The transport at the edge is assumed to be anomalous, as observed in previous experiments 12,13. A typical value of $1 \mathrm{~m}^{2} / \mathrm{s}$ is used for the design and the limiter shape is afterwards adopted for the range $0.5-2 \mathrm{~m}^{2} / \mathrm{s}$. The total heating power considered in the design was set to $5 \mathrm{MW}$ and no radiation losses are considered.

The solution of equation 1 for the design parameters is plotted in blue in figure $1 \mathrm{~b}$. The shown line represents one half of the limiter. To guarantee safe operation with smaller $D_{\perp}$ of $0.5 \mathrm{~m}^{2} / \mathrm{s}$ and for hydrogen discharges the central part was modified by inserting a parabolic part, as shown with green line in figure 1 $1 \mathrm{~b}$. To simplify mounting and to clearly separate two strike lines, the limiter shape was further modified by adding a $2 \mathrm{~cm}$ plateau in the center and a fixed linear slope at $45^{\circ}$ at the edge, the red line in the figure. 

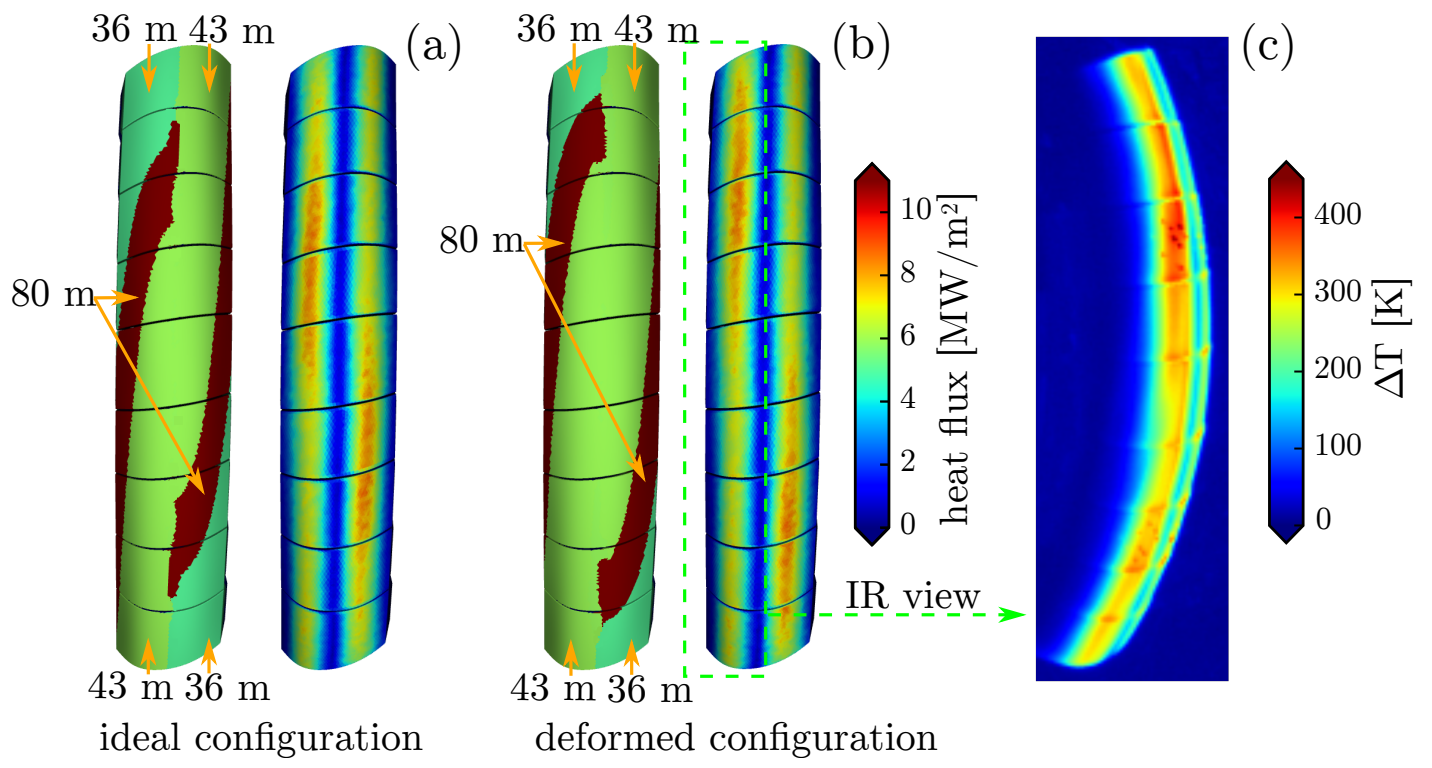

Figure 2. Connection lengths and heat flux density to the limiter. (a) - connection lengths and heat flux density for the primary limiter configuration with planar coils current of 0.13. (b) - connection lengths and heat flux density for the magnetic configuration taking into account coil deformations due to electromagnetic forces. These simulations are performed with a mimic configuration with planar coils current of 0.15 , which matches flux surface measurements. (c) - increase of the limiter surface temperature measured with an infrared camera in module 5 in a typical discharge (W7X\#20160308.007). Only one half of the limiter is visible.

This dependence was used to generate the limiter surface taking into account the $3 \mathrm{~d}$ geometry of the flux surfaces. The radial shifts were applied along local LCFS normals according to the distance to the watershed along field lines. The resulting limiter surface after smoothing was cut into 9 tiles, as presented in figure 1 . The limiter tiles are side mounted, which avoids plasma-metal interaction.

The limiter design procedure does not consider the uniformity of the power density in the vertical direction. The height of the limiters is restricted by the available support structure. As a consequence, three areas with different connection lengths are present, figure 2 2a. Field lines in the first area with $L_{c}$ about $39 \mathrm{~m}$ meet the same limiter after a single toroidal turn, in the second area with $L_{c}$ about $43 \mathrm{~m}$ the limiter in the neighbouring module after $1 \frac{1}{5}$ turns and in the third one with $L_{c}$ about $80 \mathrm{~m}$ the neighbouring limiter after $2 \frac{1}{5}$ turns. The limiter shape was produced using the radial decay length for the area with the longest connection length. The modifications of the limiter shape guarantee that the maximal allowed heat flux density on the limiter is not exceeded in the other areas.

Non-uniform connection lengths result in vertically non-uniform heat flux densities. In figure $2 a$ the heat flux densities simulated with Monte-Carlo field line diffusion model [6] for the design parameters and $P_{\text {heat }}$ of $4 \mathrm{MW}$ are presented. In this method field lines from inside the last closed flux surface are traced with an additional perpendicular 
diffusion. In figure 2 a two strike lines are visible on the left and the right sides of the watershed. The plateau insert and a large part of the linear extensions at the edges are not loaded, the maximal loads are located in the areas with the angle between the surface and the field of about $20^{\circ}$. In the strike lines a vertical structure corresponding to different connection lengths is pronounced. About $20 \%$ higher power densities are predicted in areas where the long connection zone with $L_{c}$ of about $80 \mathrm{~m}$ is located at the strike line position These areas are mainly at tiles 3 and 4 on the left and tiles 6 and 7 on the right. In agreement with the stellarator symmetry the left and right strike lines are flip symmetric: the right one is the left one rotated by 180 degrees. The power is equally partitioned between the limiters.

The field line diffusion model $[6]$ is used throughout this work to simulate limiter loads. Even though this model is not a self-consistent plasma description, its results are qualitatively consistent with the elaborated EMC3-EIRENE model [9]. In particular for the limiter case, EMC3-EIRENE simulations [10] also predict the two stellarator symmetric strike lines around the watershed and a higher loaded area at the positions defined by the long connection length. Moreover, in low density cases, i.e. when other losses can be neglected, absolute values of the heat flux density in different regions calculated with EMC3-EIRENE agree with the field line diffusion model. This agreement is not surprising, as the load distribution is in the first place determined by the field and limiter geometries. Since it is the field geometry and its modifications with error fields that are of major concern in this paper, the use of the field line diffusion model is adequate. The advantage of this model is its flexibility for testing limiter misalignments and effects of error fields.

The position of the area with longest connection length and consequently of the maximum heat flux density depends on the value of rotational transform. In the experiments the rotational transform differed from the design value because of coil deformations by the electromagnetic forces [14]. This change can be reproduced by adjusting the current in the planar coils to match the flux surface measurements. Connection lengths and simulated heat flux densities for such an adopted configuration can be found in figure $2 \mathrm{~b}$. The maximal loads are now found on tiles 2 and 3 on the left and tiles 7 and 8 on the right. This position is in agreement with the experimental observations of the left side of the limiter in module 5 with an infrared camera [18, figure 2 c. Therefore, this configuration is adopted for the remaining simulations in this paper.

The experimental data in figure 2 c show increase of the limiter surface temperature in a discharge, where the ECRH heating power was about 2.1 MW for the first $0.3 \mathrm{~s}$ and was kept between 3.8 and $3.95 \mathrm{MW}$ from $0.3 \mathrm{~s}$ until $1.2 \mathrm{~s}$. The temperature raise is about $360 \mathrm{~K}$ in the higher loaded area (tile 3 ) and about $310 \mathrm{~K}$ in the less loaded areas (tile 8). The corresponding heat flux densities calculated with the THEODOR code [19, 20] are about 4.3 and $3.5 \mathrm{MW} / \mathrm{m}^{2}$, averaged over the high heating phase. The field line diffusion model predicts values of 8.1 and $6.3 \mathrm{MW} / \mathrm{m}^{2}$ for the two areas. While the ratio of the heat flux densities in the two areas is close between the measurements and the model, the 

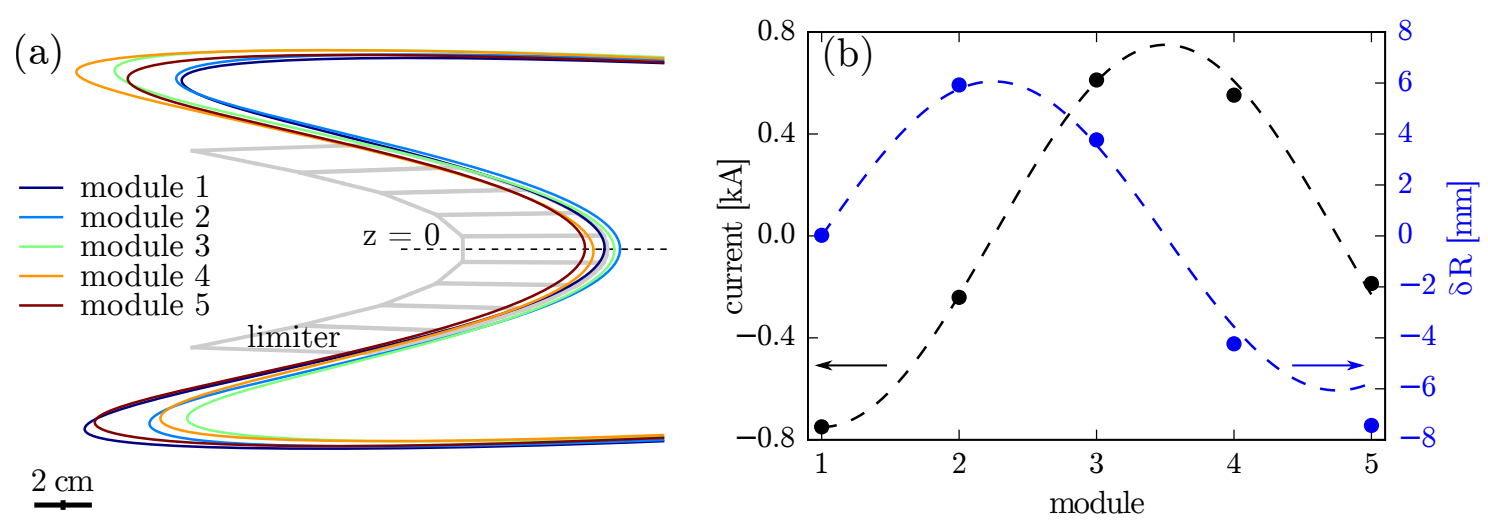

Figure 3. Perturbation of the last closed flux surface by trim coils with current distribution according to toroidal mode $n=1$. (a) - Poincaré plot in the bean shaped plane in 5 modules. Here the ideal last closed flux surface perturbed by the trim coils with amplitude of $750 \mathrm{~A}$ is shown. Different modules are shown in different colours. The scale of the plot in vertical direction is not the same as in horizontal to make the changes more visible. Limiter tiles are shown in gray. (b) - trim coil currents and the LCFS shifts relative to the ideal case. The blue dashed line is according to equation 2.

absolute values differ. The difference in the heat flux density is caused by radiative losses and by unequal partitioning of the power between the limiters. The latter follows from the analysis of the power balance in discharges with available bolometer data [5,21] and is also found later in the paper from measurements with thermocouples in the limiters. A further analysis of the infrared measurements including measurements of the heat flux radial decay length will be given elsewhere.

\section{Influence of error fields}

Distribution of the heat flux density on the limiters and partitioning of the power between the limiters can be affected by a relatively small radial perturbation of the magnetic field. Such perturbations can be due to intrinsic field errors caused by coil misalignments, magnetic materials etc. [15], or can be introduced on purpose by error field trim coils [7]. Here to study the influence of the error fields on the limiter loads the trim coils are employed both in simulations and in experiments. Five trim coils installed one per machine module can be used for both canceling the intrinsic errors or for studying their influence. In the first campaign currents in these coils were limited to $1 \mathrm{kA}$, which corresponds to relative amplitude of about $4.5 \cdot 10^{-4}$ for $\mathrm{m} / n=1 / 1$ mode. The phase of perturbations can be varied.

To illustrate the importance of the error fields for the limiter loads it is useful to consider induced deformations of the ideal last closed flux surface. For non-resonant components the amplitude of the deformation can be estimated according to [1]:

$$
\delta x=\frac{R_{0}\left|\beta_{m n}\right|}{m t+n} \cdot \frac{d x}{d r_{\mathrm{eff}}},
$$

where $R_{0}$ is the major radius, $\beta_{m n}$ is the normalized error field amplitude for the 


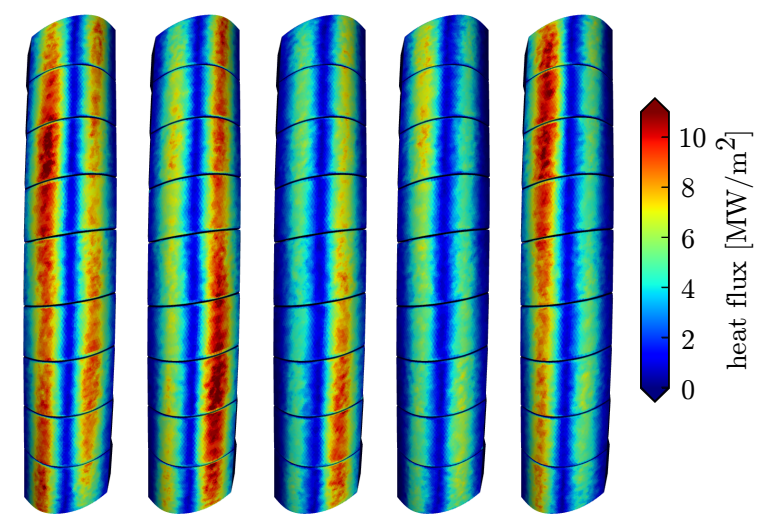

Figure 4. Simulated heat flux density to the five limiters for trim coils with $1 \mathrm{kA}$ currents in phase 5 . The phases are named by the module with the maximal current.

poloidal mode $m$ and toroidal mode $n$, and $t$ is the rotational transform. The derivative $d x / d r_{\text {eff }}$ of the observed value with respect to effective radius takes into account flux compression. Considering potential modes it can be concluded that the largest flux surface deformation is produced by $1 / 1$ mode, because the rotational transform of the W7-X configurations is close to 1 . For the limiter configuration a $1 / 1$ perturbation of $2 \cdot 10^{-4}$ is estimated to produce a helical LCFS displacement of about $4 \mathrm{~mm}$ at the limiter position. This value is about one third of the design radial decay length of $1.5 \mathrm{~cm}$, and thus a significant effect can be expected.

The expected deformation of flux surfaces by error field modes is confirmed numerically with the help of field line tracing. In figure 3 a Poincaré plot of the ideal last closed flux surface perturbed by the trim coils with a $n=1$ current distribution is presented. The flux surface is shown in five bean shaped planes corresponding to the limiter location. The ideal LCFS is seen to be considerably deformed. In some modules it is shifted into the limiter in others from the limiter. The deformation is helical as seen from the top bottom asymmetry and is consistent with the poloidal mode number $m$ of 1 .

The trim coil currents used to illustrate the LCFS deformation are given in figure 3b. Similar $n=1$ current distributions but with different amplitudes and phases are used also for other simulations and in the experiments in this work. The $n=1$ trim coil currents produce error field spectrum with the dominant $m / n=1 / 1$ mode. Other modes with $n=1$ are at least three times weaker than $1 / 1$ and decay quickly with $m$. Modes with the toroidal mode number of 2 and 3 are significantly smaller, but noticeable modes with $n=4$ and $n=6$ are present, because the number of the trim coils is limited to five. A detailed example of the error field spectrum produced with the $n=1$ trim coil currents can be found in [11]. For the present study the $m / n=1 / 1$ mode is important due to its dominant amplitude and due to the resonance denominator in equation 2. To quantify the phase and the amplitude of the LCFS deformation caused by the trim coils, it is convenient to measure the surface deviation from its ideal position at the equatorial plane, i.e. in the middle of the limiter $z=0$, figure $3 \mathrm{~b}$. The numerically calculated shifts follow the $n=1$ distribution, but the phase of the distribution is 


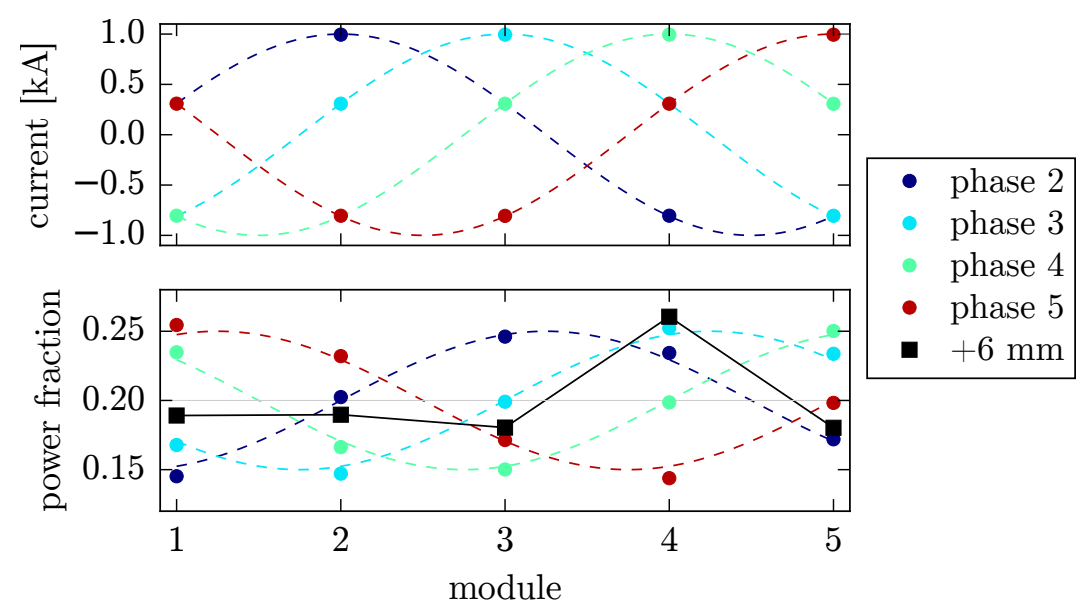

Figure 5. Simulated limiter power fractions (bottom) for different trim coil phases (top). The phases are named by the module with the maximal current. For comparison a case with ideal field but with limiter 4 shifted into the plasma by $6 \mathrm{~mm}$ is given.

shifted by $90^{\circ}$ relative to the currents in the trim coils. The amplitude of the deviation measured from field line tracing agrees with the estimation from equation 2 .

An example of simulated heat flux densities to the limiters with error fields is given in figure 4. These calculations were made for trim coil currents with amplitude of $1 \mathrm{kA}$ in phase 5 . The phases are named by the module with the maximal current, i.e. for this case the maximal current flows in the trim coil located in module 5 . The $1 / 1$ perturbation breaks both the toroidal fivefold symmetry and the poloidal pattern of the heat flux density. The ideal LCFS is most strongly displaced towards the limiter with a $90^{\circ}$ phase shift. Accordingly, the most loaded limiter is in module 1 and similarly the least loaded one is in module 4 . The left and right strike lines of a limiter are not flip symmetrical anymore. Furthermore, for two sequential limiters the strike line on one side of the first limiter is influenced in the opposite direction to the other side of the next limiter. For example, the right strike line of limiter 1 is enhanced whereas the left one of limiter 2 is weakened. Such a pattern appears because these strike lines are connected. A limiter with a beneficial shift intercepts the heat flux, whereas the connected strike line is depleted.

Distribution of the heat flux between the limiters changes with the perturbation phase. In figure 5 limiter power fractions for different trim coil phases are plotted. In agreement with the flux surface modification, the limiter with the highest fraction is in the module next to the one with the maximal trim coil current and the minimal fraction is in the preceding module. For the trim coil current of $1 \mathrm{kA}$, the amplitude of the power modification is about $5 \%$ of the total power. The ratio between the power to the most loaded limiter and the power to the least loaded one is 1.7. This ratio for individual tiles, e.g. for the left strike line on tile 5, is higher and is about 2.8.

In addition to the trim coil simulations, figure 5 also presents the case with the ideal field but with the limiter in module 4 shifted into the plasma by $6 \mathrm{~mm}$. Amplitude of power loads modification is of the same order as for the field perturbations, but the 


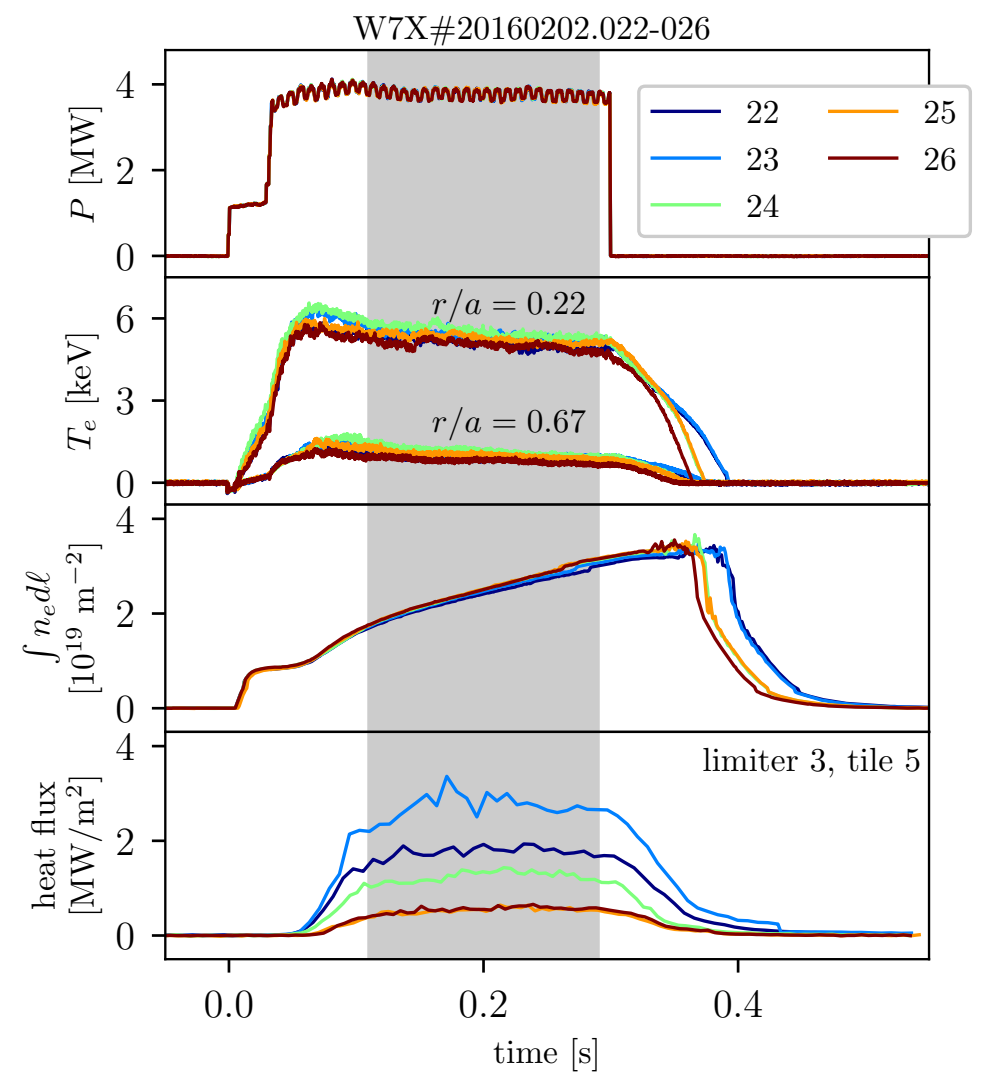

Figure 6. Plasma parameters in the experiments with the trim coil phase. The phase was sequentially changed from phase 1 to phase 5 in discharges from 20160202.022 to 20160202.026. The trim coil amplitude is $1 \mathrm{kA}$. The plots, from top to bottom, show the ECRH heating power, the electron temperature measured with ECE at two positions, the line integrated electron density and the heat flux density to tile 5 of limiter 3. The shaded area indicates the part used for the further analysis.

structure is different: power to the other modules is decreased, with the two closest modules receiving slightly less than the more distant ones.

Field perturbations can be potentially used to counteract a limiter misalignment. For the misalignment case illustrated in figure 5 the trim coil phase 5 is suitable for the correction. As it is obvious from the linear combination, a $1 \mathrm{kA}$ perturbation would decrease the fraction in module 4 , but would overload limiter 1 by the same amount. A $0.5 \mathrm{kA}$ perturbation is more suitable for this case as it equilibrates modules 1 and 4 at the half amplitude. An optimal choice of the perturbation depends on all limiter shifts, and was not actually attempted in this work.

\section{Experimental observations}

Influence of the $n=1$ field perturbations on the limiter loads was verified experimentally. Similar plasma discharges with identical fuelling and ECRH heating were performed. Only the currents in the trim coils were changed between the discharges. Two series 
of experiments were performed: phase and amplitude variations. In the first one the amplitude of the trim coil currents was fixed at $1 \mathrm{kA}$, but the phase was changed from module 1 to 5 in five steps. In the second series the phase was fixed to module 5 and the amplitude was ramped.

Limiter surface temperatures in two modules were measured with infrared cameras: in module 3 with a nearly straight view on tiles from 3 to $5(\lambda=3-5 \mu \mathrm{m})$ [16, 17], and in module 5 with an oblique view on the left half of all 9 tiles $(\lambda=8-14 \mu \mathrm{m})[18]$. These infrared data are used to reconstruct the heat flux density to the limiters with the help of THEODOR code [19,20]. In addition, limiters in modules 5 and 1 were observed with near infrared cameras $(\lambda=0.85-1 \mu \mathrm{m})$, which allowed only temperature measurements after a discharge because of the contamination from plasma radiation. Plasma wall interaction in modules 1,2,4 and 5 was monitored with fast neutral gas pressure gauges. Also $H_{\alpha}$ and $C$ II emission was measured with a sight line looking radially at limiter $3[26]$. Furthermore, temperatures of the limiter holding structure, i.e. of the $\mathrm{CuCrZr}$ tiles behind the limiters, were monitored with thermocouples in all five modules. These thermocouples are located far away from the limiter surface and can not be used for direct measurements of the surface temperature during plasma discharges. Nevertheless, the data from thermocouples in different limiters can be applied to verify the power distribution between limiters, as shown later in the paper.

Typical plasma parameters of the experiments are shown in figure 6 for one series of discharges. In this set, the trim coil amplitude was fixed at $1 \mathrm{kA}$, but the phase was sequentially changed from phase 1 to phase 5 . For the analysis a part of the discharges is used, where the limiter loads are relatively stable and which is highlighted in the figure with shaded area. The ECRH heating power is about $3.75 \mathrm{MW}$ with an additional modulation of $\pm 0.1 \mathrm{MW}$. The modulation was used for transport related studies and is not relevant for the present discussion. The central electron temperature measured with the ECE diagnostic [23] is about $6 \mathrm{keV}$, and the line integrated electron density measured with the dispersion interferometer 24$]$ is on average about $2.5 \cdot 10^{19} \mathrm{~m}^{-2}$. The line integrated density is still increasing, but this has no visible effect on the limiter power loads. These plasma parameters are typical for the first W7-X campaign. The plasma is in the electron root regime with highly peaked electron temperature, flat ion temperature profile with central temperature between 1 and $2 \mathrm{keV}$ and flat electron density profile [5].

The discharges used to study the effect of the trim coils are reproducible, figure 6. The central electron temperature and the line integrated electron density vary by about $5 \%$ from shot to shot, whereas the edge electron temperature is the same within about 10$15 \%$. At the same time, the power distribution to the limiters changes significantly for different trim coil phases, as demonstrated with the heat flux density to tile 5 of limiter 3 in the figure. These limiter data are summarized also in figures 7 and 8 .

Measured power densities to tiles 3, 4 and 5 of limiter 3 are compared with the simulations for different perturbation phases in figure 7. As a reference, a case without trim coils is shown in the first column. The applied perturbation phase for each column is 


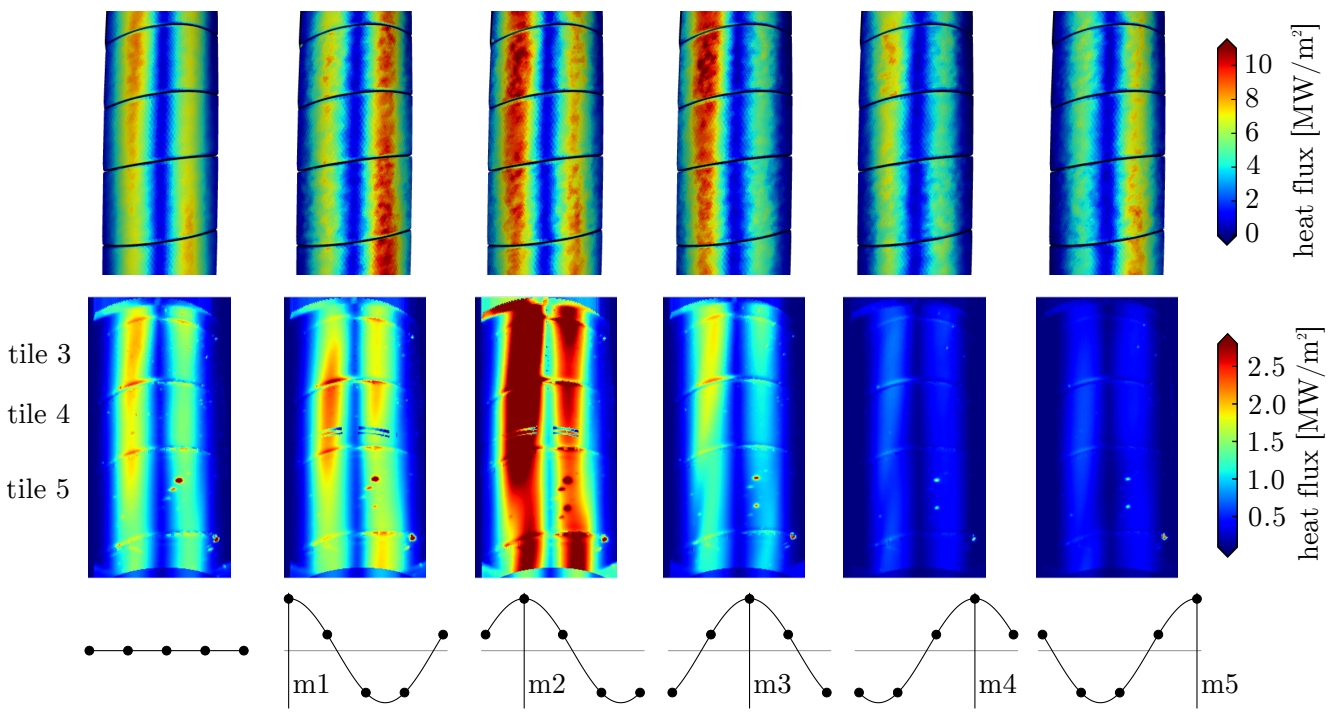

Figure 7. Comparison of the simulated (top) and measured (middle) heat flux densities to tiles 3 to 5 in module 3 for different trim coil phases phases (bottom). Trim coil experiments with variation of the phase at constant amplitude of $1 \mathrm{kA}$. The experimental data are averaged over the phase with flat top limiter loads.

given in the last row. The experimental data are averaged over the phase with flat top limiter loads. The experimental data confirm that the limiter loads are affected by the error fields and that the change for a given limiter depends on the phase of the mode. The observed phase behaviour qualitatively agrees with the expectations and with the simulations. Both the simulations and the measurements show the maximum load to limiter 3 for the trim coil phase 2, slightly increased loads for phase 1 , and reduced loads for phase 4. On the other hand it is also observed that for phase 5 the loads to limiter 3 are reduced stronger than expected. The absolute values of the measured data are discussed below.

Similar results for different perturbation phases are obtained for limiter 5. Measured power deposited to the left side of tiles 1, 5 and 9 of limiter 5 are shown in figure 8 . Here the experimental data are plotted with symbols and the lines shown least square fits. The fit results indicate a phase shift between the tiles. The phase shift is caused by the helical nature of the perturbation and is consistent with poloidal mode number $m$ of 1 . For comparison also the power deposited to tile 5 of limiter 3 and measurements of $H_{\alpha}$ in module 3 are shown. All experimental data consistently confirm the phase variation. The absolute values of power densities measured in the experiments are well below the values in the simulations. This is explained by still changing plasma stored energy, charge exchange losses and radiated power losses, where the plasma radiation is estimated to be dominant. For discharges with the heating power above about $1 \mathrm{MW}$, the radiated power fraction is between 20 and $40 \%$, as measured with a bolometer system at the end of the first campaign [25]. The experiments with the trim coils took place in the early stage, where data from the bolometer system are not available. But the plasma radiation is expected to be even higher, because the machine conditioning was 


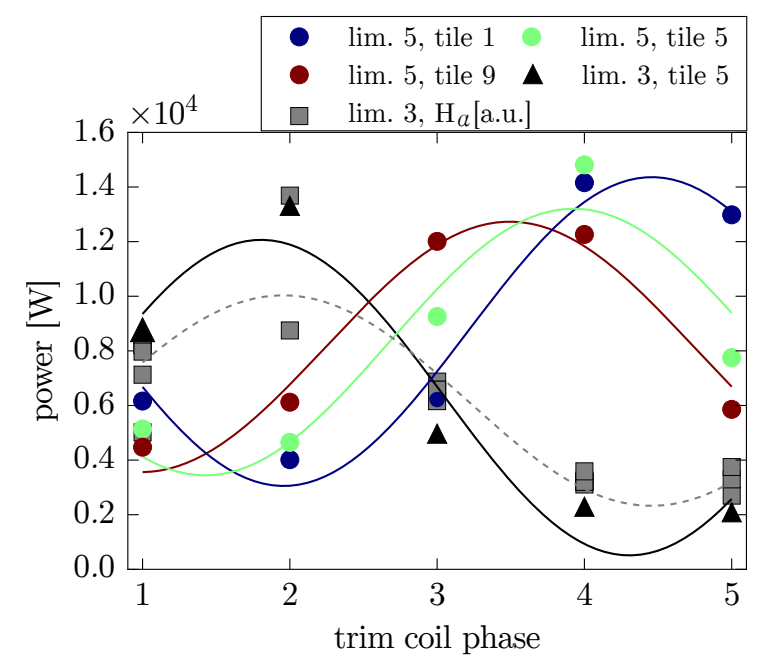

Figure 8. Variation of the deposited power and amplitude of $H_{\alpha}$ signal with trim coil phase. The shown signals are: power deposited to left side of tiles 1,5 and 9 in module 5 , power deposited to tile 5 in module 3 . The $H_{\alpha}$ signal is measured in module 3. The trim coil phase is counted in modules. The experimental data are shown with symbols. The lines are cosine fits to the experimental data.

improving during the whole campaign [5]. The results of the field line diffusion model can be scaled with the actual power going to the limiters.

In addition to the absolute scale, the modulation of the power with the trim coil phase in the experiments is larger than predicted by the model. The ratio of the maximal power to the minimal power, figure 8, is about 3.5 for limiter 5 and is about 6.5 for limiter 3, whereas the field line diffusion model predicts a ratio of 2.8. This is likely caused by the intrinsically present asymmetry of the power distribution between limiters. This asymmetry, caused either by limiter misalignments or internal field errors, is discussed below. It is probably for the same reason that in the reference case without perturbations in figure 7 the experimental data do not seem to show the flip symmetry between left and right sides.

Influence of the perturbation field scales with the amplitude, as expected from equation 2. In figure $9 \mathrm{a}$ the power deposited to different tiles of limiters 3 and 5 is shown. In these experiments the phase of the trim coils is fixed to phase 5 . For limiter 5 an up down asymmetry is observed. The power to tile 1 of limiter 5 increases, whereas the power to tile 9 decreases. For the middle tiles, e.g. for tile 5, and also for the whole limiter on average the power remains roughly constant. The asymmetry is qualitatively consistent with the simulations, figure 4. For limiter 3 a decrease of the power is observed for all tiles and for both the left and the right strike lines. The reduction of the power to limiter 3 is also confirmed by spectroscopic measurements. The scalings of the observed signals with the trim current amplitude are close to linear.

In figure 9b the neutral gas pressure distribution is shown for different perturbation amplitudes in phase 5. Fast pressure gauges are available in all modules, with exception of module 3. Without trim coils the pressure is always peaked in module 4 . The reason 

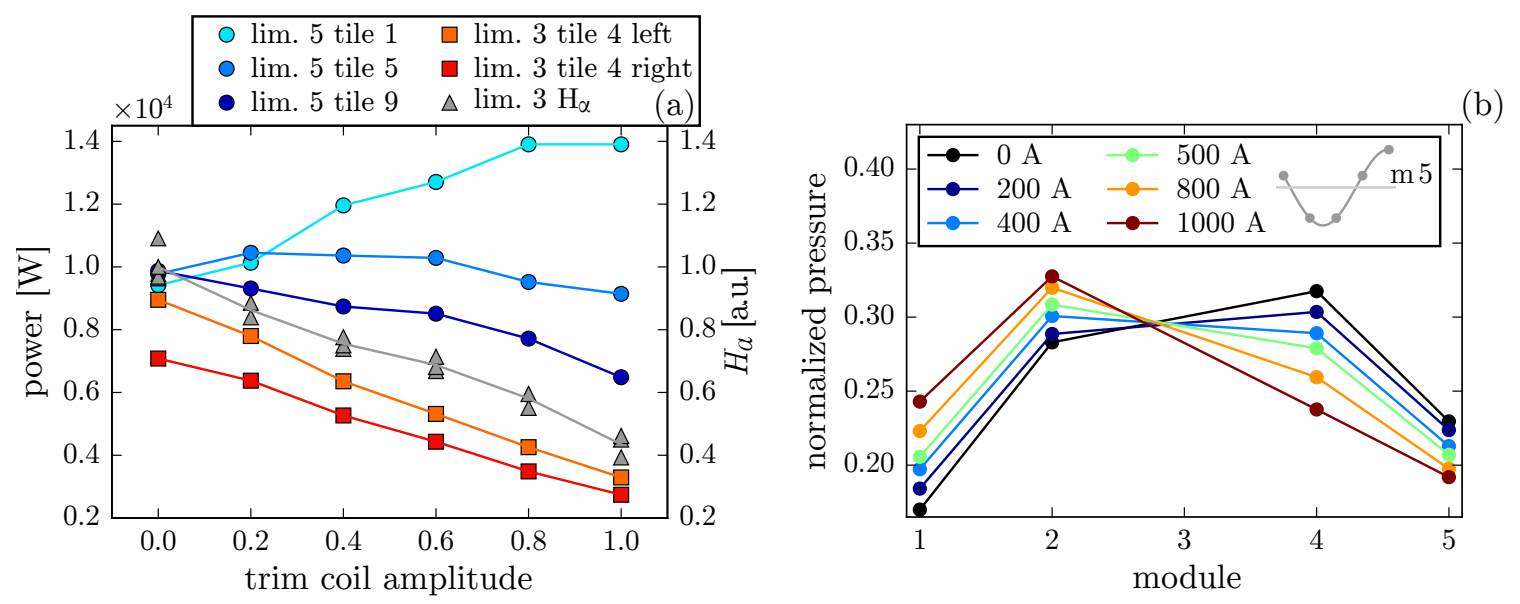

Figure 9. Scaling of the trim coil effect with the current amplitude for fixed phase 5. The experimental data are averaged over the phase with flat top limiter loads. (a) dependence of the power deposited to different tiles of limiters 3 and 5 as measured with infrared cameras. Also amplitude of $H_{\alpha}$ signal at limiter 3 is given. (b) - dependence of the neutral pressure distribution. Fast pressure gauges are available in all modules, with exception of module 3 .

for this is unclear, but is not likely to be caused solely by the plasma-limiter interaction in this module. For the used trim coil phase the neutral pressure in module 4 is reduced, and on the opposite side of the machine in module 1 the pressure is increased. The change of the neutral pressure increases with the perturbation amplitude. These changes are consistent with the expected redistribution between the limiters, figure 5 . A direct measurement of the power fractions for all limiters was not possible because of the limited number of infrared systems. To characterize the power partitioning measurements of the temperature in the limiter holding structure are of interest. Temperatures of $\mathrm{CuCrZr}$ tiles behind the limiters were monitored with thermocouples in all five modules. Such measurements have a large inertia but can give an approximation for the total absorbed energy. Usually the temperatures gradually increase during plasma experiments and return to the initial values in the night, figure $10 \mathrm{a}$. The maximal values achieved at the end of an experimental day can be considered as a proxy for the absorbed energy. Similarly, the peak difference of the temperature normalized by the sum of the differences for all modules is representative of the power partitioning between the limiters. In figure $10 \mathrm{~b}$, the temperature fractions are compared for experimental days with and without trim coil operation. The trim coil data include three experimental days, where almost all discharges were performed with trim coils in phase 5 and with amplitude of $1 \mathrm{kA}$. In agreement with the other experimental data and with the simulations, the fractions to limiters 3 and 4 are reduced and the fraction for limiter 1 is increased.

The thermocouple measurements of the temperatures indicate an intrinsic asymmetry of the power distribution between the limiters even without trim coils, figure 10p. Limiter in module 1 receives the highest fraction of about 0.28 , whereas the power fraction 

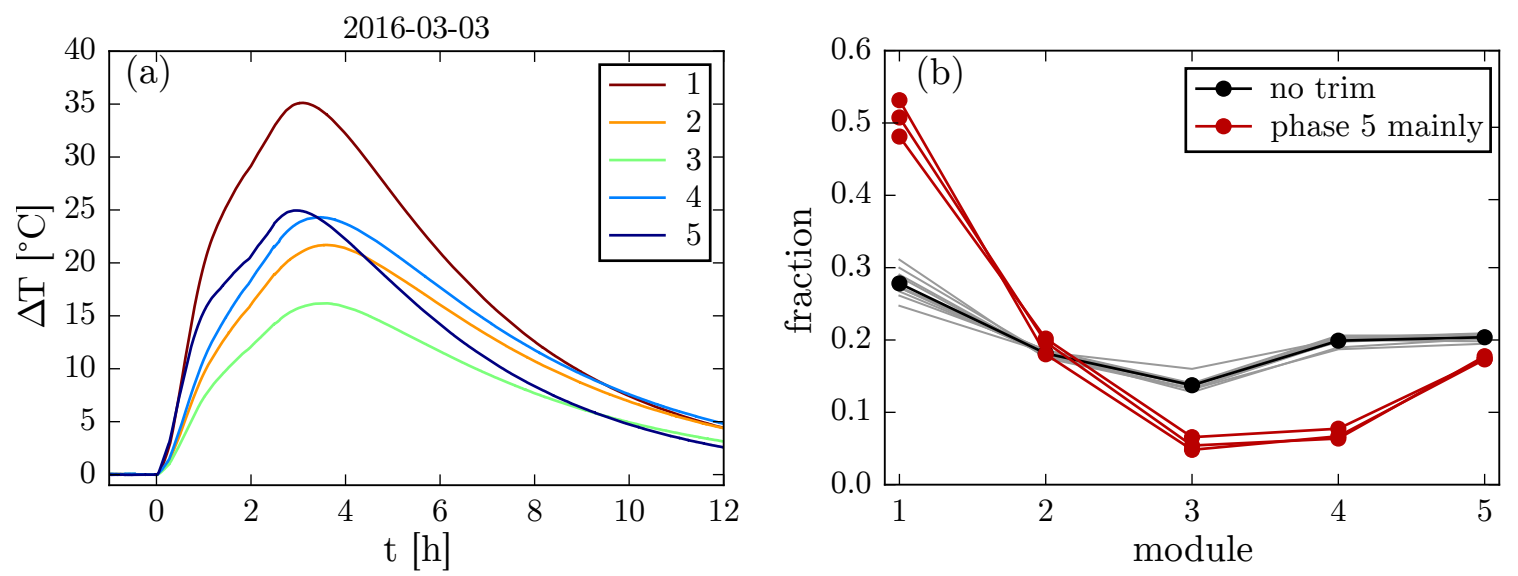

Figure 10. Temperatures in the limiter holding structure. (a) - time evolution of the temperature difference for an experimental day without trim coils. (b) - fractions $\Delta T_{i} / \sum_{i} \Delta T_{i}$ for values at the end of experimental days with and without trim coil operation. Individual experimental days without trim coils are plotted with thin lines, the average value is given with thick line. Values with trim coils include three experimental days, where almost all discharges were performed with $1 \mathrm{kA}$ phase 5 .

for the least loaded limiter in module 3 is about 0.14 . These values are somewhat higher than those discussed for the simulations of the error field effects due to $1 \mathrm{kA}$ trim coils and of the limiter misalignment by $6 \mathrm{~mm}$, see figure 5 . It is not possible to conclude about the source of the asymmetry from the shape of the distribution in figure 10 b. Independent measurements of the internal error fields with the flux surface mapping technique [14] are planned for future experiments.

The observation of the intrinsic asymmetry by the thermocouples is supported by further evidences. For discharges with a low radiation fraction, i.e. where the most of the power is deposited to the limiters, the global power balance is deficient with up to $40 \%$, if toroidal fivefold symmetry is assumed [5,21]. Near infrared cameras show higher post-discharge temperatures of the limiter in module 1. Also, the post-campaign inspection of the limiters shows a different deposition pattern for limiter 1 as compared to the other modules [17]. This asymmetry can be caused by a misalignment of the limiters or an intrinsic $n=1$ error field. Symmetrization of the loads with the help of the trim coils was not attempted because the limiter performance was not a limiting factor in the experiments. Symmetrization of power loads can be of higher importance for the later divertor operations.

\section{Conclusions}

In the first campaign Wendelstein 7-X was operated in a limiter configuration [4]. The magnetic configuration was set up to have no large islands in the scrape-off layer. Five poloidal limiters were installed stellarator symmetrically. In an ideal situation the power 
losses are equally distributed between the limiters. This configuration allowed first successful W7-X experiments [5].

The limiter shape was designed for a smooth distribution of the heat flux over the surface. Two stellarator symmetric strike lines are present on each limiter. The strike lines are not uniform vertically. Each strike line has three areas with different connection lengths and as a consequence with different incident power densities. The highest power density on the limiter is observed for the area with connection length of about $80 \mathrm{~m}$. The overall structure of the strike lines is confirmed experimentally with the infrared camera measurements.

The heat flux to the limiters can be significantly changed by $1 / 1$ error field of the order of $10^{-4}$. In this paper the influence of $n=1$ field perturbations on the limiter loads was studied numerically with field line tracing [6]. Poincaré plots show a sufficiently large helical shift of the ideal last closed flux surface, as compared to the radial heat flux decay length. For example, for the primary limiter magnetic configuration used in the first W7-X campaign a $1 / 1$ perturbation of $2 \cdot 10^{-4}$ produces a displacement of about $4 \mathrm{~mm}$. This value is about one third of the design radial decay length of $1.5 \mathrm{~cm}$. The amplitude of the deformation scales linearly with the error field, the phase is shifted by $90^{\circ}$ relative to the currents in the trim coils.

Field line diffusion model [6] used to simulate the heat fluxes predicts a break of both the fivefold toroidal and poloidal flip symmetry. The power distribution between the limiters follows the $n=1$ dependence with the same phase shift relative to the perturbation currents as the deformation of the last closed flux surface. The left and right strike lines are not flip symmetric anymore. For two sequential limiters the strike line on one side of the first limiter is influenced in the opposite direction to the other side of the next limiter. Such a pattern appears because these strike lines are connected.

The influence of $1 / 1$ error field on the limiter loads is confirmed experimentally with a number of diagnostics, which includes infrared cameras [16, 18], neutral pressure gauges, thermocouples in the limiter mounting structure, spectroscopic measurements. The error fields were superimposed with five trim coils and similar plasma discharges were performed. The power deposited to a limiter is found to strongly depend on the phase of the perturbation. The maximal loads are measured for the trim coils phase with maximal current in the previous module and the minimal loads for the phase with maximal current in next module. This is consistent with the simulations and the expected phase shift. A change of the poloidal structure of the strike lines is observed. A phase shift between top and bottom parts of a limiter is measured, and this shift is consistent with the poloidal mode number $m$ of 1 . The effect of the error fields scales with the perturbation amplitude. In general, the experimental observations are qualitatively consistent with the simulations. There are also noticeable experimental differences in the poloidal structure of the heat flux density, which can be due to plasma effects like radiation, due to limiter installation errors, or due to intrinsic error fields.

Even without the trim coils the power distribution between the limiters is observed to be asymmetric. Direct measurements of the power fractions for all limiters was not possible 
because of the limited number of infrared systems. The evidences for the asymmetry include measurements of the temperature of the limiter holding structure, near infrared measurements of limiter post-discharge temperatures, deficiency of the global power balance with the symmetry assumption [5,21] and a post-campaign visual inspection of the deposition patterns [17]. The asymmetry can be due to a misalignment of the limiters or due to error fields. An $1 / 1$ error field of the order of $10^{-4}$ is expected [15] but is still to be verified [22]. A $n=1$ trim coil configuration can be potentially used to counteract both sources. A $1 \mathrm{kA}$ current distribution produces a disturbance with an amplitude about the same as a limiter shift of about $6 \mathrm{~mm}$. In the first campaign the load symmetrization was not a vital issue because of a large radiated power fraction [25] This results are, however, of interest for the later campaigns, where similar questions might be of higher importance. It is worth mentioning that a limiter misalignment and error fields result in different structures of the heat flux density.

\section{Acknowledgment.}

This work has been carried out within the framework of the EUROfusion Consortium and has received funding from the Euratom research and training programme 20142018 under grant agreement No 633053. The views and opinions expressed herein do not necessarily reflect those of the European Commission.

\section{References}

[1] G. Grieger, C. Beidler et al., "Physics studies for helical-axis advanced stellarators", Proc. 12th Int. Conf. Plasma Physics and Controlled Nuclear Fusion Research, Nice (1988) vol. 2, pp 369 $-387$

[2] J. Kißlinger, C. Beidler et al., "Island divertor for the stellarator Wendelstein 7-X", Proc. of 21st European Conf. Controlled Fusion and Plasma Physics, Montpellier, France (1994) vol 18B, part 1, pp 36871

[3] H. Renner, J. Boscary et al., "The capabilities of steady state operation at the stellarator W7-X with emphasis on divertor design", Nuclear Fusion 40, 1083 - 1093 (2000); http: //dx.doi.org/10.1088/0029-5515/40/6/306

[4] T.S. Pedersen, T. Andreeva et al., "Plans for the first plasma operation of Wendelstein 7-X", Nuclear Fusion 55, 126001 (2015); http://stacks.iop.org/0029-5515/55/i=12/a=126001

[5] R.C. Wolf, A. Ali et al., "Major results from the first plasma campaign of the Wendelstein 7-X stellarator", Nuclear Fusion 57, 102020 (2017); http://stacks .iop.org/0029-5515/57/i=10/ $\mathrm{a}=102020$

[6] S.A. Bozhenkov, J. Geiger et al., "Service oriented architecture for scientific analysis at W7-X. An example of a field line tracer." Fus. Eng. Des. 88, 2997 - 3006 (2013); http://dx.doi.org/10. 1016/j.fusengdes.2013.07.003

[7] T. Rummel, K. Riße et al., "The Trim Coils for the Wendelstein 7-X Magnet System", IEEE Trans. Applied Superconductivity 22, 4201704 (2012); http://ieeexplore.ieee.org/xpls/icp.jsp? arnumber $=6117068$

[8] S.A. Bozhenkov, F. Effenberg et al., "Limiter for the early operation phase of W7-X", 41st EPS Conf. on Plasma Physics, Berlin, Germany (2014), P-1.080; http://ocs.ciemat.es/ EPS2014PAP/pdf/P1.080.pdf 
[9] Y. Feng, F. Sardei et al., "3D Edge Modeling and Island Divertor Physics", Contributions to Plasma Physics 44, 57-69 (2004); http://dx.doi.org/10.1002/ctpp.200410009

[10] F. Effenberg, Y. Feng et al., "Numerical investigation of plasma edge transport and limiter heat fluxes in Wendelstein 7-X startup plasmas with EMC3-EIRENE" Nuclear Fusion 57, 036021 (2017) http://stacks .iop.org/0029-5515/57/i=3/a=036021

[11] S.A. Bozhenkov, S.A. Lazerson et al., "Methods for measuring 1/1 error field in Wendelstein 7-X stellarator", Nuclear Fusion 56, 076002 (2016); http://stacks.iop.org/0029-5515/56/i=7/ $\mathrm{a}=076002$

[12] M. Hirsch, J Baldzuhn et al., "Major results from the stellarator Wendelstein 7-AS", Plasma Physics and Controlled Fusion 50, 053001 (2008); http://stacks.iop.org/0741-3335/50/i= $5 / \mathrm{a}=053001$

[13] H. Maassberg, R Brakel et al., "Transport in stellarators", Plasma Physics and Controlled Fusion 35, B319 (1993); http://stacks.iop.org/0741-3335/35/i=SB/a=026

[14] M. Otte, D. Aßmus er al., "Setup and initial results from the magnetic flux surface diagnostics at Wendelstein 7-X", Plasma Physics and Controlled Fusion 58, 064003 (2016); http://stacks. iop.org $/ 0741-3335 / 58 / i=6 / a=064003$

[15] T. Andreeva, T. Bräuer et al., "Tracking of the magnet system geometry during Wendelstein 7-X construction to achieve the designed magnetic field", Nuclear Fusion 55, 063025 (2015); http://stacks.iop.org/0029-5515/55/i=6/a=063025

[16] G. A. Wurden, L. A. Stephey, et. al., " A high resolution IR/visible imaging system for the W7-X Limiter", Rev. Sci. Instrum. 87, 11D607 (2016); http://dx.doi.org/10.1063/1.4960596

[17] G.A. Wurden, C. Biedermann et al., "Limiter observations during W7-X first plasmas", Nuclear Fusion 57, 056036 (2017); http://stacks .iop.org/0029-5515/57/i=5/a=056036,

[18] H. Niemann, M. W. Jakubowski et al., "Power loads in the limiter phase of Wendelstein 7-X", 43rd EPS Conf. on Plasma Physics, Leuven, Belgium (2016), P4.005; http://ocs.ciemat.es/ EPS2016PAP/pdf/P4.005.pdf

[19] A. Herrmann, W. Junker et al., "Energy flux to the ASDEX-Upgrade diverter plates determined by thermography and calorimetry" Plasma Physics and Controlled Fusion 37, 17 (1995); http://stacks.iop.org/0741-3335/37/i=1/a=002

[20] A. Hermann and ASDEX Upgrade team, "Limitations for divertor heat flux calculations of fast events in tokamaks", 28th EPS Conf. on Plasma Physics, Madeira, Portugal (2001), P5.104; http://www.cfn.ist.utl.pt/EPS2001/fin/pdf/P5.104.pdf

[21] S.A. Bozhenkov, G. Fuchert et al., "Power Balance Analysis of Wendelstein 7-X Plasmas Using Profile Diagnostics", 43rd EPS Conf. on Plasma Physics, Leuven, Belgium (2016), O2.106; http://ocs.ciemat.es/EPS2016PAP/pdf/02.106.pdf

[22] S A. Lazerson, M. Otte et al., "Error field measurement, correction and heat flux balancing on Wendelstein 7-X", Nuclear Fusion 57, 046026 (2017); http://stacks.iop.org/0029-5515/57/ $i=4 / a=046026$,

[23] M. Hirsch, R. Burhenn et al., Microwave and Interferometer Diagnostics for Wendelstein 7-X, 41st EPS Conf. on Plasma Physics, Berlin, Germany (2014), P1.069; http://ocs.ciemat.es/ EPS2014PAP/pdf/P1.069.pdf

[24] J. Knauer, P. Kornejew et al., "New Dispersion Interferometer for the Stellarator Wendelstein 7X", 43rd EPS Conf. on Plasma Physics, Leuven, Belgium (2016), P4.017; http://ocs.ciemat. es/EPS2016PAP/pdf/P4.017.pdf

[25] D. Zhang, R. Burhenn et al., Investigation of the Radiative Power Loss in the Limiter Plasmas of W7-X, 43rd EPS Conf. on Plasma Physics, Leuven, Belgium (2016), P4.015; http://ocs. ciemat.es/EPS2016PAP/pdf/P4.015.pdf

[26] M. Krychowiak, A. Adnan et al. "Overview of diagnostic performance and results for the first operation phase in Wendelstein 7-X", Review of Scientific Instruments 87, 11D304 (2016) http://aip.scitation.org/doi/abs/10.1063/1.4964376 